\title{
LOS VALORES CULTURALES Y EL DESARROLLO SOCIOECONÓMICO: UNA COMPARACIÓN ENTRE TEORÍAS CULTURALES*
}

\author{
María Ros
}

Universidad Complutense de Madrid

\begin{abstract}
RESUMEN
Las teorías transculturales de Hofstede (1980), Schwartz (1994) Triandis (1995) e Inglehart (1998) representan en la actualidad las formulaciones más contrastadas sobre las dimensiones culturales de valores. A pesar del interés y popularidad del modelo de Inglehart, no existen trabajos que traten de analizar la validez convergente y discriminante de esta teoría con relación a otras teorías culturales. Este trabajo trata de llenar este hueco comparando la dimensión de Postmaterialismo de Inglehart con las dimensiones culturales de Autonomía, Conservación, Jerarquía, Igualitarismo, Armonía y Competencia de Schwartz. Para lo cual seguimos varios procedimientos. En primer lugar, estudiamos la relación entre las puntuaciones en las dimensiones culturales de ambos modelos con una muestra de 40 países. A continuación, relacionamos las dimensiones culturales convergentes con variables externas, macrosociales y macroeconómicas, de estas mismas sociedades. Finalmente, analizamos el impacto de la cultura, medida por los valores, y del nivel socioeconómico previo sobre el desarrollo económico y el desarrollo social de las mismas sociedades. Los resultados indican que la similaridad conceptual entre las dos teorías se produce entre las dimensiones culturales del Postmaterialismo, Autonomía Intelectual y Afectiva e Igualitarismo; que los valores son resultado de una serie de condiciones macroeconómicas y macrosociales. Así, mientras que el crecimiento económico refuerza los valores materiales, el desarrollo humano refuerza valores más solidarios. Ahora bien, la orientación de valores explica gran parte del desarrollo económico y del desarrollo humano posterior.
\end{abstract}

* Trabajo presentado en la Mesa de Psicología Social, Sesión 3, «La dimensión sociocultural en psicología social: una nueva convergencia hacia la perspectiva sociológica», coordinada por María Ros, VII Congreso Nacional de Sociología, Salamanca, 20-22 de septiembre de 2001. 


\section{INTRODUCCIÓN}

Existen varias teorías sobre la estructura de los valores culturales que se usan para entender las similitudes y diferencias en las sociedades contemporáneas. Las teorías transculturales de Hofstede (1980), Schwartz (1994), Triandis (1996) e Inglehart (1998) representan en la actualidad las formulaciones más contrastadas sobre las dimensiones culturales de valores. Las cuatro teorías comparten un interés por encontrar dimensiones con un significado común que permita comparar las sociedades contemporáneas. Se diferencian en los instrumentos que usan, en las muestras que utilizan y en los supuestos estructurales de sus modelos. La teoría de Hofstede considera que cuatro dimensiones culturales son suficientes para entender las variaciones culturales: individualismo, distancia de poder, masculinidad y evitación de la incertidumbre. Schwartz postula la existencia de seis tipos de valores culturales: Jerarquía, Igualitarismo, Conservación, Autonomía, Armonía y Competencia. Triandis argumenta que la dimensión de individualismo y colectivismo debe combinarse con la igualdad o desigualdad en las relaciones sociales dando lugar a cuatro tipos de valores culturales, individualismo horizontal e individualismo vertical, y colectivismo horizontal y colectivismo vertical, que se estructuran en dos dimensiones bipolares, mientras que Inglehart considera que el cambio cultural se explica sobre la base de la dimensión de Materialismo-Postmaterialismo y la dimensión de Modernización-Postmodernización.

Se han producido varios trabajos que han tratado de entender las similitudes y diferencias entre ambas. En este sentido, la teoría cultural de Hosfstede ha sido relacionada con la teoría cultural de Schwartz (Schwartz, 1994). La dimensión cultural de Individualismo de Hofstede $(\mathrm{H})$ está relacionada con Autonomía e Igualitarismo de Schwartz $(\mathrm{SH})$ y se opone a Conservación, lo cual refuerza la idea de que se puede encontrar un énfasis cultural en el desarrollo de la persona como agente autónomo y que de forma voluntaria trabaja por ayudar a los demás como opuesto a alguien insertado en el mantenimiento del status quo; la dimensión Distancia de Poder $(\mathrm{H})$ está vinculada a Conservación, Jerarquía y Competencia $(\mathrm{SH})$ y se opone a la dimensión cultural del Individualismo, lo cual implica que la dimensión de Desigualdad Social es lo opuesto al Individualismo; Evitar la Incertidumbre $(\mathrm{H})$ está relacionada, aunque de forma poco intensa, con Armonía, Igualitarismo, Competencia, Autonomía $(\mathrm{SH})$ y, por último, Masculinidad $(\mathrm{H})$ se relaciona con Competencia y Jerarquía (SH).

Recientemente, Gouveia y Ros (2000) han analizado las similaridades y diferencias entre la dimensión del Individualismo y Distancia de Poder del modelo de Hofstede y la dimensión cultural de Autonomía y Conservación de Schwartz con una muestra de 20 países. Los autores han concluido que la dimensión cultural del Individualismo de Hosfstede está significativamente relacionada con la dimensión cultural de Autonomía de Schwartz y se opone a la dimensión de Distancia de Poder, que a su vez está relacionada con Conser- 
vación. Esto supone una réplica de los resultados de Schwartz (1994). Pero, además, encontraron que mientras que el Individualismo de Hosfstede está asociado a un individualismo competitivo por su relación con el producto interior bruto, la dimensión cultural de la Autonomía de Schwartz se encuentra más vinculada al índice de desarrollo humano.

A pesar del interés y popularidad del modelo de Inglehart, no existen trabajos que traten de analizar la validez convergente y discriminante de esta teoría con relación a otras teorías culturales. Nuestro objetivo en este trabajo es tratar de llenar este hueco comparándola con la teoría cultural de Schwartz. Para lo cual seguiremos varios procedimientos. En primer lugar, estudiaremos la relación entre las puntuaciones en las dimensiones culturales de ambos modelos con una muestra de 40 países; esta estrategia nos permitirá hallar las comunalidades y diferencias entre ambas teorías. A continuación, relacionaremos las mismas dimensiones culturales con variables externas (como indicadores macrosociales y macroeconómicos) de las mismas sociedades en que se han medido sus valores culturales para verificar su validez convergente y discriminante. Finalmente, analizaremos el impacto de la cultura, medida por los valores, y del nivel socioeconómico previo sobre el desarrollo económico y el desarrollo social de las mismas sociedades.

Pero, antes de desarrollar estos objetivos, pasemos a exponer cada una de las teorías.

\section{LA TEORÍA DE LOS VALORES CULTURALES DE SCHWARTZ}

Treinta y cinco mil personas agrupadas en 122 muestras correspondientes a 49 países, entre 1988 y 1993, contestaron de forma anónima el cuestionario de valores, el SVS, de Schwartz (1992) en sus lenguas nacionales. Evaluaron la importancia de 56 valores "como principios-guía en MI vida". Cada uno de los valores estaba seguido en paréntesis de una pequeña frase explicativa (ej., «igualdad», igualdad de oportunidades para todos). Las respuestas podían variar desde 7 (de máxima importancia), a 3 (importante), a 0 (no importante) y a -1 (opuesto a mis valores). Un análisis multidimensional de cada uno de los 56 valores dentro de cada uno de los países estableció la equivalencia conceptual de 45 de los 56 valores (Schwartz, 1992, 1994a). Estos 45 valores fueron los que entraron en los análisis SSA para la obtención de las dimensiones culturales.

La teoría considera que las dimensiones culturales de los valores reflejan los temas básicos o los problemas que las sociedades deben afrontar para regular la actividad humana. Según Schwartz, tomándolo de Kluckhön (1951), estos problemas básicos son: la naturaleza de las relaciones entre el individuo y el grupo, garantizar un comportamiento responsable entre los ciudadanos que ayude a preservar las sociedades y mantener la relación de la especie humana con la naturaleza y el mundo social. 
Para analizar la solución de estos tres problemas sociales básicos distingue siete tipos de valores culturales que se estructuran en torno a tres dimensiones bipolares:

- Conservación versus Autonomía. Esta dimensión sirve para distinguir las soluciones culturales que se han dado a la relación conflictiva entre individuo y grupo. El polo de Conservación agrupa las culturas en que la persona es vista como una entidad que está incluida en la colectividad, mientras que el polo de Autonomía caracteriza a las culturas en las que la persona es percibida como un ente autónomo que encuentra significado en su propia diferencia. Según la teoría, las culturas que puntúan alto en Conservación otorgan importancia al mantenimiento del status quo, y restringen las acciones que puedan interferir en la solidaridad grupal o en el orden tradicional. Valores como orden social, respeto por la tradición, seguridad familiar o sabiduría son ejemplos de algunos valores que configuran esta dimensión. La Autonomía se bifurca en Autonomía Intelectual y Afectiva. Las culturas que puntúan alto en Autonomía otorgan énfasis cultural a las ideas y el pensamiento de los individuos; valores como ser curioso, abierto de miras o creativo tienen en común compartir estos aspectos. La Autonomía Afectiva representa el énfasis cultural para que los individuos persigan las experiencias afectivas positivas; valores como placer, vida variada y vida excitante, comparten estas experiencias afectivas.

Esta dimensión también ha sido denominada individualismo-colectivismo (Hofstede, 1980; Kim et al., 1994).

- Jerarquía versus Igualitarismo. Esta dimensión refleja las formas en que las culturas socializan a los individuos para que consideren el bienestar de los demás y manejen las interdependencias sociales. Las sociedades potencian que las personas atiendan a las diferencias de poder, los sistemas jerárquicos de roles para asegurar el comportamiento de responsabilidad social. Así, las culturas altas en Jerarquía consideran legítima la distribución desigual de poder, roles y recursos; valores como poder social, autoridad, humildad y riqueza forman este valor tipo. Como contraste, las culturas altas en Igualitarismo dan una solución alternativa que consiste en considerar a los miembros de la sociedad como seres iguales que comparten intereses como seres humanos. En estas culturas se socializa a sus miembros a comprometerse voluntariamente a cooperar con los demás y a preocuparse de su bienestar. Valores como justicia social, libertad, responsabilidad, honestidad, pertenecen a este polo de la dimensión.

- Competencia versus Armonía. Esta dimensión refleja las soluciones culturales al problema de las relaciones del ser humano con la naturaleza. Estas soluciones van desde dominar y cambiar el mundo de forma activa a aceptar el mundo como es tratando de encajar en él. El tipo de valor de Competencia resume el énfasis cultural por salir adelante a través de la autoafirmación; por eso, valores como ambicioso, tener éxito, ser competente o asumir riesgos forman parte de esta dimensión. Mientras que el polo de la Armonía resume el énfasis cultural por el encaje armónico de las personas con el ambiente. Los 
valores de unidad con la naturaleza, proteger el medio ambiente y mundo de belleza comparten esta motivación armónica.

Estas tres dimensiones culturales bipolares representan la estructura de las incompatibilidades culturales entre tipos de valores. La teoría también postula una estructura de relaciones culturales de compatibilidad. Jerarquía y Conservación se relacionan positivamente porque comparten la idea del actor social (individual o grupal) insertado en una colectividad de seres humanos interdependientes y mutuamente obligados entre sí. Igualitarismo y Autonomía se relacionan positivamente debido a que comparten la idea de actor social como una entidad autónoma. Competencia se relaciona positivamente con los valores de Autonomía, ya que ambos presumen la legitimidad de cambiar el status quo y ambos enfatizan la actividad estimulante. Competencia y Jerarquía se relacionan positivamente porque los esfuerzos por salir adelante a menudo son a costa de otros y dan lugar a una distribución desigual de roles y recursos que se justifican por la legitimidad de las diferencias jerárquicas. Armonía es compatible con los valores de Conservación, por compartir el interés por evitar el cambio, y con Igualistarismo, con quien comparte interés por las relaciones cooperativas.

Esta teoría ha sido relacionada con dimensiones del comportamiento laboral, como la centralidad del trabajo, las normas sociales sobre el trabajo y los valores asignados al trabajo (Schwartz, 1999). Más recientemente, también se ha usado como variable antecedente junto a variables socioeconómicas como el PIB, inflación, violación de derechos humanos, desigualadades de ingresos. Y se ha estudiado la relación de estas variables con las preocupaciones personales y sociales, indicadores del bienestar subjetivo. Culturas altas en Igualitarismo y Armonía se preocupan menos de los problemas personales y más de los problemas sociales y mundiales. Mientras que el patrón inverso ocurre con culturas altas en Jerarquía. Mucho del impacto objetivo que tienen las condiciones socioeconómicas sobre las preocupaciones personales y sociales está mediado por las orientaciones culturales. Igualitarismo cultural, Armonía y la violación de derechos humanos fueron los predictores más consistentes de la intensidad relativa de las preocupaciones sociales frente a las personales. De los tres, el Igualitarismo cultural es el de mayor influencia y el impacto de la violación de derechos humanos está mediado por el Igualitarismo (Schwartz y Melach, 2000).

\section{LA TEORÍA DE LOS VALORES CULTURALES DE INGLEHART}

«Por cultura entendemos un sistema de actitudes, valores y comportamientos compartidos ampliamente por la sociedad y transmitidos de generación en generación. Mientras la naturaleza humana es biológicamente innata y universal, la cultura es aprendida y varía de una sociedad a otra» (Inglehart, 1998, p. 18). 
Inglehart, entre 1973 y 1990, midió las prioridades valorativas de muestras representativas de 43 sociedades a través de la Encuesta Mundial de Valores. Doce metas, presentadas en tres grupos de cuatro ítems, fueron diseñadas para evaluar las prioridades materialistas y postmaterialistas. Un total de 54.743 personas de estas sociedades respondieron tanto a sus prioridades valorativas como a una serie de actitudes hacia la familia, el trabajo, la salud y el bienestar subjetivo.

Análisis factoriales realizados sobre las prioridades en valores materialistas y postmaterialistas en cada una de las 43 sociedades revelaron la existencia de una dimensión cultural formada por dos polos, Materialismo y Postmaterialismo:

- Materialismo. Las culturas que densan alto en este factor se caracterizan por respaldar valores como "apoyar fuerzas armadas poderosas", "combatir la subida de precios», "luchar contra la delincuencia», «mantener el orden» y «mantener una economía estable».

- Postmaterialismo. Las culturas que puntúan alto en este factor apoyan valores como «tener una sociedad menos impersonal», «más participación en el trabajo», "más participación en el gobierno», "las ideas cuentan más que el dinero», «libertad de expresión» y «ciudades más bonitas».

Según Inglehart (1998), la rotación factorial proporciona una única dimensión en que los ítems materialistas tienen correlaciones positivas y los ítems postmaterialistas correlaciones negativas con el factor. Los análisis factoriales realizados en cada uno de los países encuestados replicaron los resultados en este factor, con la excepción del valor "ciudades más bonitas», que fue excluido del índice transnacional de Postmaterialismo porque en algunos países estaba relacionado con los ítems materialistas y en otros con los postmaterialistas. Se construyó un índice transnacional de los valores materialistas/postmaterialistas basado en los nueve ítems que tienen polaridad coherente en el nivel global, asignando polaridad neutra a los dos ítems que hacen referencia a la economía.

Inglehart, transponiendo en el orden social la jerarquía de motivaciones que Maslow postula para el nivel personal, señala en 1977 que las sociedades tendrán motivaciones de orden superior (ej., autoexpresivas) cuando tengan resueltas necesidades de orden inferior (ej., de supervivencia o de seguridad). Por eso, las condiciones económicas de las sociedades son responsables de sus orientaciones valorativas. La escasez económica en el ámbito societario genera inseguridad y, para superarla, es funcional tener prioridades materialistas, mientras que el bienestar económico genera seguridad y, por tanto, contribuirá al desarrollo de orientaciones valorativas postmaterialistas. El cambio de valores en el ámbito social desde el Materialismo hacia el Postmaterialismo es, por tanto, consecuencia del cambio en las condiciones económicas de las sociedades, de menor bienestar a mayor bienestar económico.

En 1998, Inglehart agrega una segunda dimensión, ya tradicional, de modernización cuyo origen procede de Weber e introducida en Psicología 
Social por Inkeles y Smith (1974), que sirve para analizar el paso de las sociedades tradicionales a las sociedades modernas. Denomina a esta segunda dimensión Modernización, que implica el cambio de la autoridad religiosa a la autoridad estatal, a través del proceso de secularización y de burocratización. En síntesis, dos dimensiones, la tradicional versus racional-legal y la dimensión de supervivencia (Materialismo) versus bienestar subjetivo (Postmaterialismo), dan cuenta del $51 \%$ de la variación transnacional tanto en valores como en actitudes y comportamientos religiosos, sociales, políticos y económicos. Siendo la segunda dimensión más importante que la primera, ella sola explica el $30 \%$ de la varianza transnacional. Si más de la mitad de la varianza transnacional viene explicada por estas dos dimensiones, esto implica que el resto de la varianza se puede deber a factores no cubiertos por estas dimensiones y a aspectos no comunes, sino específicos, de las sociedades analizadas.

La relación entre el desarrollo económico de los países y las dos dimensiones culturales muestra que los países más ricos (Norte de Europa, Asia, países anglosajones, Europa Occidental) comparten una cultura de autoridad racional secular y postmaterialistas, mientras que los países más pobres (Sur de Asia, África y Latinoamérica) están más cerca de la autoridad tradicional y de valores de supervivencia, y las sociedades de la Europa Oriental se caracterizan por los valores materialistas y por dar una importancia considerable a la autoridad estatal en lugar de la tradicional. Ahora bien, la relación entre desarrollo económico y aumento de los valores postmaterialistas puede deberse o bien a la influencia del nivel educativo actual del entrevistado o bien al impacto que la seguridad familiar le proporcionó en sus años formativos, medido por el nivel de educación y de ocupación de sus padres cuando el entrevistado era niño. Según Inglehart (1998), en los análisis realizados sobre seis países, Alemania, Países Bajos, Austria, EE.UU., Italia y Finlandia, los coeficientes de regresión de la seguridad formativa explican más varianza, 0,63 de media, que el nivel educativo de los encuestados, 0,26 de media.

$\mathrm{El}$ autor vincula estos cambios a los cambios experimentados en las condiciones económicas de la modernización y la postmodernización. La teoría de la modernización propone que el desarrollo económico está unido a cambios en cierto modo predecibles en la cultura y en la vida social y política. Estos cambios, unidos a la industrialización, constituyen un síndrome que consiste en: la especialización ocupacional, la burocratización, la centralización, la urbanización, la educación formal de masas, el desarrollo de los medios de comunicación de masas, la secularización, el aumento de los empresarios y de las motivaciones empresariales, la cadena de montaje de producción en masa y el surgimiento del Estado moderno, así como creencias y valores que apoyan las altas tasas de crecimiento económico.

Por tanto, ampliando la teoría clásica de la modernización, Inglehart (2000) sugiere que el desarrollo económico da lugar a dos dimensiones psicosociales de las diferencias transculturales: una primera dimensión unida a la industrialización temprana y la emergencia de la clase trabajadora, y una 
segunda dimensión que refleja las condiciones de bienestar económico de las sociedades industriales avanzadas con el incremento de los sectores de servicios y de conocimiento (Bell, 1973). Mientras que las sociedades preindustriales se caracterizan por valorar la tradición (respeto a la jerarquía, obediencia, orgullo nacional) y la seguridad (económica y física, desconfianza hacia los demás), las industriales se diferencian porque se cambia la tradición por la racionalidad, se mantiene la seguridad y aparece la secularización, y, por último, las sociedades postindustriales tienden a tener valores opuestos y así enfatizan los valores seculares racionales y los valores autoexpresivos y se cuestiona la racionalidad de la modernidad como valor absoluto.

Además, un conjunto de actitudes y comportamientos están asociados a estas dos dimensiones. Las sociedades que enfatizan valores de supervivencia o materialistas muestran: «niveles bajos de bienestar subjetivo, tienen mala salud, escasa confianza interpersonal, relativa intolerancia con exogrupos, bajo apoyo a la igualdad de género, niveles altos de confianza en la ciencia y tecnología, bajos en activismo medioambiental y relativamente favorables a gobiernos autoritarios» (Inglehart, 2000, p. 28). En este sentido, Diener y Diener $(1995 a)$ encuentran una relación importante $(r=0,65)$ entre el PIB de los países y el bienestar subjetivo. Las sociedades que enfatizan valores postmaterialistas o autoexpresivos muestran un perfil diferente: «Mayor bienestar subjetivo, mayor interés en la protección del medio ambiente, más apoyo a los movimientos a favor de la igualdad de derechos para la mujer, lesbianas, homosexuales y extranjeros, así como mayor participación en las decisiones económicas y políticas" (Inglehart, 2000, p. 27).

En síntesis, Inglehart sugiere una modificación en la teoría de la modernización en el sentido de que en esta teoría hay una relación lineal con el crecimiento económico durante el proceso de industrialización, tal y como apuntaban Inkeles y Smith (1974); después se llega a un umbral donde se satisfacen las necesidades básicas y, a partir de este umbral, los valores de la modernización dejan de reforzarse y se comienzan a reforzar los valores del Postmaterialismo.

\section{EL ESTUDIO}

Nuestro objetivo es comparar los modelos de los valores culturales de Schwartz e Inglehart para ver su validez convergente y discriminante. Existen dos procedimientos de comparación de modelos. Un procedimiento consiste en correlacionar directamente los constructos de las teorías entre sí para distinguir las coincidencias y diferencias entre ellos. Otro procedimiento consiste en relacionar los modelos con variables externas a ellos para verificar su validez convergente y divergente. En principio, si las dimensiones teóricas miden lo mismo se relacionarán de forma similar con estas variables externas y, de lo contrario, se relacionarán de forma distinta. En nuestro caso utilizaremos ambos procedimientos. 
Para aplicar el primer procedimiento, correlacionaremos las puntuaciones de 40 países en los valores del Postmaterialismo de Inglehart con las puntuaciones de estos mismos países en las dimensiones culturales de la teoría de Schwartz extraídas en torno a los mismos años.

\section{Comparación conceptual entre las teorias}

En línea con la definición conceptual de las dimensiones culturales en los dos modelos y con la relaciones estructurales de ambas teorías, nuestra hipótesis es que la dimensión de Postmaterialismo (I) estará positivamente relacionada con el valor cultural del Igualitarismo $(\mathrm{SH})$ por su énfasis común en la igualdad de derechos de la mujer, libertad de expresión y la tolerancia hacia los exogrupos.

Por tanto, esta dimensión estará negativamente relacionada con Jerarquía (SH) ya que este valor cultural implica un respaldo a las desigualdades sociales de roles, así como sumisión hacia las figuras de autoridad.

También consideramos que existe una relación plausible entre el Postmaterialismo (I) y la Autonomía Intelectual $(\mathrm{SH})$ ya que ambas dimensiones comparten los valores de autoexpresión de la persona, curiosidad intelectual, así como de libertad, para decidir sobre las cuestiones que les conciernen. El Postmaterialismo también estará positivamente relacionado con la dimensión de Autonomía Afectiva $(\mathrm{SH})$ por compartir los valores de disfrutar y de tener una mayor calidad de vida.

Dado que en la teoría de Schwartz la dimensión de Autonomía se opone a la de Conservación, esperamos que el Postmaterialismo (I) se oponga a los valores de Conservación (SH) por su énfasis en la tradición y seguridad.

Finalmente, creemos que los valores del Postmaterialismo (I) estarán negativamente relacionados con los valores de Competencia ( $\mathrm{SH}$ ), ya que éstos enfatizan la transformación activa del mundo a través de los logros económicos. Sin embargo, esperamos que el Postmaterialismo (I) se relacione positivamente con los valores de Armonía (SH) porque ambos comparten una preocupación por el medio ambiente.

\section{Desarrollo económico y cambio cultural}

Existen pocos trabajos transculturales que aborden los determinantes del cambio social medido como cambio en las condiciones económicas. MacClelland (1961) fue uno de los primeros autores que, siguiendo la tesis weberiana, analizó el impacto de la modernización sobre las orientaciones motivacionales del individuo, motivación de logro, y de éstas sobre el desarrollo económico. Estudió el desarrollo económico de 18 naciones modernas y el nivel de motivación de logro expresado en los libros que se leían en los colegios públicos de 
estas naciones. Los resultados mostraron que las naciones altas en motivación de logro presentaban mayores porcentajes de desarrollo económico. Posteriormente, este mismo autor verificó que esta relación estaba mediada por el efecto indirecto que la educación tenía sobre el desarrollo. Más recientemente, Hofstede (1991) ha relacionado las condiciones económicas con los valores del individualismo. Correlaciona condiciones económicas antecedentes con los valores del individualismo unos años más tarde y estos mismos valores con condiciones económicas posteriores. El autor encuentra que ambas relaciones eran significativas pero que el desarrollo económico es más responsable del individualismo que éste del desarrollo económico.

La teoría de Inglehart también destaca que la influencia va de las condiciones económicas a los valores y no a la inversa. Por tanto, es de esperar que los valores postmaterialistas estén allí donde haya habido mayor desarrollo económico previo. El autor usa datos transversales de la tasa de crecimiento económico de un país desde 1965 a 1990 y muestra una correlación de 0,44 con la importancia dada a la autoridad racional-secular, según datos de los años 9091. Para medir la relación entre valores y desarrollo económico correlaciona el PIB per cápita de los países en 1988 con los valores postmaterialistas de estos mismos países entre 1990-91 y encuentra una correlación de 0,66 p’<0,0001 (Inglehart, 1998, p. 199). Díez Nicolás (1994) lo hace relacionando la renta per cápita del año 1962 de las regiones españolas con su nivel de Postmaterialismo entre 1989-92 y encuentra una correlación aún más alta $(r=0,83)$. Las diferencias entre ambos estudios parecen sugerir que la relación entre seguridad económica previa y Postmaterialismo posterior es más factible encontrarla en periodos largos de tiempo, y que esta relación es más intensa cuando el marco estatal común permite la igualación de otros factores que podrían influir en la relación, como la orientación del sistema educativo y, más en general, las orientaciones en política económica.

Inglehart (1998) analiza también el efecto contrario, la influencia de los valores sobre el desarrollo económico. Para lo cual mide el impacto que el Postmaterialismo, la motivación de logro, la tasa de educación primaria y secundaria y la inversión tienen sobre la tasa media de crecimiento económico. Muestra que tres variables, la motivación de logro, la tasa de educación primaria en los años sesenta y el PIB per cápita en los sesenta, explican el 70\% del crecimiento económico. Según estos datos, el Postmaterialismo no contribuye al crecimiento económico.

Por tanto, parece que el Postmaterialismo funciona de forma diferente si analizamos riqueza per cápita que cuando analizamos crecimiento económico. Indagar en los antecedentes de esta situación será uno de los objetivos de nuestro trabajo. Con este fin, analizaremos el peso relativo de los valores culturales sobre el cambio económico y sobre el cambio en desarrollo humano producido en los países en un periodo de diez años. Para lo cual realizamos dos análisis de regresión. En el primero relacionamos el efecto del PIB per cápita del año 1985 y de los valores culturales sobre el PIB per cápita del año 1995. En el 
segundo comparamos el efecto del IDH del año 1985 y de los valores culturales sobre el IDH de 1995. De este modo podremos diferenciar el efecto de los valores culturales y del nivel previo de riqueza sobre el nivel de riqueza alcanzado diez años más tarde. De modo similar, trataremos de diferenciar el efecto de los valores culturales y del nivel previo de desarrollo humano sobre el nivel de desarrollo humano alcanzado diez años después.

\section{Dimensiones culturales y variables macroeconómicas y macrosociales}

La teoría de Inglehart ha sido relacionada con indicadores económicos, PIB e índices de crecimiento económico, pero no con indicadores macrosociales como IDH. Sólo la dimensión cultural de Autonomía de Schwartz ha sido relacionada a la vez con variables macrosociales y macroeconómicas (Gouveia y Ros, 2000). Nuestro objetivo es relacionar esta teoría con el resto de las dimensiones culturales.

En esta dirección hemos seleccionado los indicadores macrosociales y macroeconómicos que tenían mayor asociación con las variables culturales de la teoría de Inglehart y Schwartz.

Variables macroeconómicas: PIB, producto interior bruto per cápita. Es un indicador general de la riqueza de un país. El PIB del 85, en el estudio, tiene un rango de 276 a 16.844 dólares. El PIB del 95 tiene un rango de 1.422 a 24.881 dólares. Crecimiento económico es la diferencia en el PIB país a país entre dos periodos económicos ${ }^{1}$.

Variables macrosociales: Indice de Desarrollo Humano (IDH). Es un indicador de la distribución del desarrollo humano per cápita de un país. El IDH del 85 está formado por tres indicadores: Expectativa de vida, Conocimientos (alfabetización de adultos) e Ingresos (PIB per cápita). Su rango va de 30 a 99, lo cual significa que en el primer caso falta un $70 \%$ para que ese desarrollo llegue a todo el mundo, mientras que en el segundo caso sólo falta un $1 \%$. El IDH del 95 está formado por los siguientes indicadores: Longevidad (compuesto de la esperanza media de vida y la tasa de mortalidad de niños pequeños), Conocimientos (compuesto por la tasa de alfabetización de adultos y la matriculación escolar en primaria y secundaria) y Nivel de vida decente, expresado por el PIB. Su rango va de 45 a $96^{2}$.

\section{Variables culturales:}

Postmaterialismo = índice formado por la diferencia de la puntuación de un país en la media de los ítems postmaterialistas menos los ítems materialistas ${ }^{3}$. Los

Datos extraídos del Informe del Banco Mundial.

2 Datos extraídos del Informe del Desarrollo Humano, años 1990 y 1998.

3 Se usó un índice aditivo basado en la suma de los cinco ítems postmaterialistas y otro índice aditivo de los cuatro ítems materialistas, dejándose como neutros ciudades más bonitas y los dos ítems que hacen referencia a la economía (Inglehart, 1998, p. 171). 
ítems postmaterialistas son "tener una sociedad menos impersonal», "más participación en el trabajo», "más participación en el gobierno», «las ideas cuentan más que el dinero» y «libertad de expresión». Los ítems materialistas son «apoyar fuerzas armadas poderosas», "luchar contra la delincuencia», "mantener el orden» y «apoyar el crecimiento económico». El índice es el resultado de restar a los ítems postmaterialistas los materialistas. Este índice tiene un rango de 7 a 33 .

Autonomía Intelectual = índice formado por la media del país en la puntuación dada a los siguientes valores: "creatividad", "ser curioso" y "de mente abierta». El rango de esta variable va de 3,89 a 5,40.

Autonomía Afectiva = índice formado por la media del país en la puntuación dada a los siguientes valores: "vida variada», "vida excitante», "placer», «disfrutar de la vida». El rango de puntuaciones va de 2,69 a 4,31.

Igualitarismo = índice formado por la media del país en la puntuación dada a los siguientes valores: «igualdad», «ser leal», «libertad», «responsable», «ser honesto», «justicia social», «ayudar» $\mathrm{y}$ «mundo en paz». El rango de puntuaciones va de 4,32 a 5,38.

Jerarquía = índice formado por la media del país en la puntuación dada a los siguientes valores: "poder social», «riqueza», "autoridad», «influyente»y «humilde». El rango de puntuaciones va de 1,58 a 3,70.

Conservación = índice formado por la media del país en la puntuación dada a los valores de «seguridad familiar», "respeto por la tradición», "orden social», "moderado», "limpio», "honra a sus padres», "seguridad nacional», "reciprocidad de favores», «autodisciplinado», "devoto», «sabiduría», «obediente», «educado", "saber perdonar», "proteger la imagen pública». El rango de puntuaciones va de 3,05 a 4,27 .

Armonía = índice formado por la media del país en la puntuación dada a los valores de "unidad con la naturaleza», "proteger el medio ambiente» y «mundo de belleza». El rango de puntuaciones va de 3,70 a 4,90.

Competencia $=$ índice formado por la media del país en la puntuación dada a los valores de "capaz», "tiene éxitos», "elegir sus metas», «independiente», «ambicioso». El rango de puntuaciones va de 3,53 a 4,73.

\section{Muestra:}

Hemos seleccionado aquellos países que tienen datos comparables en Postmaterialismo, Autonomía Intelectual y Afectiva, Igualitarismo, Conservación, Jerarquía y Armonía. De los 43 países que participaron en el estudio de Inglehart y de Schwartz coinciden 40. Los países seleccionados para este estudio, en orden alfabético, son: Alemania Occidental, Alemania Oriental, Argentina, Austria, Bélgica, Brasil, Bulgaria, Canadá, Corea del Sur, República Checa, Chile, China, Dinamarca, Eslovaquia, Eslovenia, España, Estados Unidos, Estonia, Francia, Finlandia, Georgia, Grecia, Holanda, Hungría, India, Inglaterra, Irlanda, Italia, Japón, Macedonia, Méjico, Noruega, Polonia, Portugal, Rumania, Rusia, Sudáfrica, Suecia, Suiza, Turquía ${ }^{4}$.

\footnotetext{
${ }^{4}$ Se han usado los datos de los países publicados en Inglehart (1998) y Schwartz (1999).
} 


\section{Procedimiento:}

Se seleccionaron los países que aparecen publicados tanto en los estudios de Inglehart (1998) como de Schwartz (1994), y se computaron sus respectivas puntuaciones en cada una de las dimensiones culturales. Los datos de las dimensiones culturales se extrajeron en ambos casos en torno a los años noventa.

Los datos correspondientes a las dimensiones macroeconómicas y macrosociales fueron extraídos de los Informes anuales del Banco Mundial, entre los años 85 y 97 .

\section{Analisis de datos:}

Se usó el paquete estadístico SPSS para analizar los datos. Se obtuvieron tanto estadísticos descriptivos como medias y correlación de cada una de las dimensiones culturales con variables macroeconómicas y macrosociales. También se usó el análisis de regresión entre variables macroeconómicas y macrosociales y variables culturales.

\section{RESULTADOS}

\section{Comparación empirica entre las teorias}

La tabla 1 muestra la relación entre los valores culturales del modelo de Inglehart y del de Schwartz.

\section{TABLA 1}

Correlación entre los tipos culturales de Inglehart y Schwartz

\begin{tabular}{|c|c|c|c|c|c|c|c|}
\hline \multirow[b]{2}{*}{ Schwartz } & \multicolumn{3}{|l|}{ Inglehart } & \multicolumn{3}{|c|}{ Schwartz } & \multirow[b]{2}{*}{$\begin{array}{l}\text { A. Inte- } \\
\text { lectual }\end{array}$} \\
\hline & $\begin{array}{c}\text { Postmate- } \\
\text { rialismo }\end{array}$ & Armonia & $\begin{array}{l}\text { Conser- } \\
\text { vación }\end{array}$ & $\begin{array}{l}\text { Jerar- } \\
\text { quía }\end{array}$ & $\begin{array}{c}\text { Compe- } \\
\text { tencia }\end{array}$ & $\begin{array}{l}\text { A. Afec- } \\
\text { tiva }\end{array}$ & \\
\hline 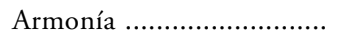 & 0,18 & & & & & & \\
\hline Conservación .................... & $-0,58^{* *}$ & $-0,38^{*}$ & & & & & \\
\hline Jerarquía .......................... & $-0,31$ & $-0,70^{* *}$ & $0,60^{* *}$ & & & & \\
\hline Competencia ...................... & $-0,21$ & $-0,48^{* *}$ & 0,10 & $0,53^{* *}$ & & & \\
\hline Autonomía Afectiva ......... & $0,50^{* *}$ & 0,003 & $-0,80^{* *}$ & $-0,23$ & 0,11 & & \\
\hline Autonomía Intelectual ..... & $0,55^{* *}$ & $0,48^{* *}$ & $-0,82^{* *}$ & $-0,51^{* *}$ & $-0,30$ & $0,61^{* *}$ & \\
\hline Igualitarismo ........................ & $0,67^{* *}$ & 0,27 & $-0,59^{* *}$ & $-0,41^{*}$ & $-0,04$ & $0,50^{* *}$ & $0,37^{*}$ \\
\hline
\end{tabular}

${ }^{*} p^{\prime}<05 ;{ }^{* *} p^{\prime}<01$.

El Postmaterialismo está positiva y significativamente relacionado con Igualitarismo y Autonomía Intelectual y Afectiva. Lo cual significa que los paí- 
ses que puntúan alto en Postmaterialismo también puntúan alto en Igualitarismo y Autonomía Intelectual y Afectiva. Es más, el Postmaterialismo está negativamente relacionado con Conservación y con Jerarquía, tal y como predeciría la teoría de los valores culturales de Schwartz; también se encuentra negativamente relacionado con Competencia, pero su relación con Armonía no es tan intensa como con Autonomía e Igualitarismo, en contra de lo que habíamos pronosticado. Este resultado puede atribuirse a que Inglehart quitó el ítem «ciudades más bellas» del índice de Postmaterialismo ${ }^{5}$, con lo cual no queda ningún ítem relacionado directamente con la preocupación con el medio ambiente y, por tanto, su relación con Armonía es poco intensa.

En esta misma tabla también aparecen las correlaciones entre los valores culturales de la teoría de Schwartz. Podemos ver si se cumplen los supuestos estructurales de la teoría. Las relaciones de incompatibilidad se cumplen: Armonía tiene una relación significativa y negativa de $r=-0,48\left(p^{\prime}<0,01\right)$ con Competencia; Conservación se relaciona de forma negativa y significativa con Autonomía Intelectual $\left(r=-0,82 ; p^{\prime}<01\right)$ y con Autonomía Afectiva $\left(r=-0,80 ; p^{\prime}<0,01\right)$, y, por último, Jerarquía está negativa y significativamente relacionada con Igualitarismo $\left(r=-0,41 ; p^{\prime}<0,05\right)$ y Autonomía Intelectual $\left(r=-0,51 ; p^{\prime}<0,01\right)$. Estas relaciones son similares a las encontradas por Schwartz (1994), excepto entre Competencia y Conservación, que aquí aparecen débil pero positivamente relacionados y que en Schwartz (1994) están negativamente vinculados.

Por otra parte, también se cumplen las compatibilidades propuestas por la teoría: Jerarquía y Conservación $(r=0,60)$; Igualitarismo y Autonomía Intelectual $(r=0,50)$ y Autonomía Afectiva $(r=0,37)$; Competencia y Jerarquía $(r=0,53)$; y Armonía e Igualitarismo $(r=0,27)$. Estas relaciones de compatibilidad son en nuestro caso similares pero más intensas que las encontradas por Schwartz (1994). Hay dos relaciones de compatibilidad que no se cumplen, entre Competencia y Autonomía Intelectual $(r=-0,30)$ y entre Armonía y Conservación $(r=-0,38)$, que tampoco se cumplían en Schwartz (1994). Es necesario señalar que existen diferencias entre el conjunto de países comparado en Schwartz (1994) y el conjunto de países comparado en este trabajo. En el trabajo de Schwartz se compararon 22 países, los que eran comunes al conjunto de países analizados por Hofstede, mientras que en este estudio se han comparado 40. Las diferencias más importantes son que en nuestro estudio están menos representadas las culturas asiáticas y autralianas y están más representadas las culturas europeas, tanto la occidental como la oriental (entre ambas suman 14 países que no estaban en Schwartz, 1994), así como Latinoamérica. Por tanto, es posible que la incompatibilidad entre Competencia y Autonomía Intelectual se deba al mayor peso del número de países europeos, ya que estu-

5 Se realizó por razones de identidad de significado de la dimensión del Postmaterialismo entre culturas. Análisis factoriales realizados en los países menos desarrollados, como India, entre otros, mostraron que este ítem estaba vinculado al Materialismo. 
dios previos (Ros y Schwartz, 1995) han mostrado que los países de la Europa Occidental son altos en Autonomía Intelectual y Afectiva pero bajos en Competencia. Pero esta conjetura debería ser contrastada en estudios que contengan un conjunto más equilibrado de países procedentes de las distintas culturas.

Los resultados sobre las incompatibilidades y compatibilidades entre las dimensiones nos permiten concluir que ya no es posible seguir hablando del individualismo y el colectivismo en el ámbito cultural como bloques monolíticos, en el sentido que lo usaban Hofstede (1980) o Triandis (1990). Parece necesario distinguir entre un individualismo Competitivo, que se caracteriza por el énfasis en los valores de transformar activamente el contexto en beneficio propio, y un individualismo basado en la Autonomía de la persona, es decir, preocupado por el desarrollo en el ser humano de la creatividad, la exploración de nuevas ideas, la apertura de mente y de disfrute de la vida. De forma similar, también tenemos que empezar a diferenciar entre un colectivismo preocupado por mantener la jerarquía social y la conservación del status quo y un colectivismo que enfatiza el trato similar a todos los seres humanos junto a una preocupación por su bienestar social, ya que enfatiza los valores de igualdad, libertad, justicia social. Desde el punto de vista de Triandis (1996, 1998) hablaríamos, por tanto, de dos Individualismos, uno Vertical que reforzaría la competitividad, similar tanto a la dimensión cultural de Competencia, y otro Horizontal relacionado con la autoconfianza, similar a la Autonomía Intelectual y Afectiva, y de dos Colectivismos, uno Vertical relacionado con la integridad familiar, similar a los valores culturales de Jerarquía y Conservación, y otro Horizontal relacionado con la interdependencia y la sociabilidad, similar al Igualitarismo de Schwartz.

La posibilidad de distinguir entre estos tipos de individualismo y de colectivismo ha encontrado apoyo en dos estudios anteriores. Ros y Schwartz (1995) analizan el perfil cultural de Europa Occidental con relación al resto del mundo. El perfil de prioridades en Europa Occidental no concuerda con la dicotomía clásica de contraste del individualismo-colectivismo. La cultura europea occidental es claramente individualista en un sentido pero no en otro. Su énfasis en los tipos de valor que asumen una visión de la persona como un ser autónomo y voluntario como base de la unidad social la convierten en individualista. Sin embargo, la cultura europea occidental también enfatiza la preocupación por los demás, antes que el egoísmo, la riqueza, el poder social o la ambición. Las políticas del Estado de bienestar resumen el respaldo de sus ciudadanos a los valores de solidaridad. En una dirección similar, Schwartz y Ros (1996) comparan el perfil de valores de los países de Europa Occidental con EE.UU. y muestran que ambos comparten valores individualistas y colectivistas aunque de distinto signo. En Europa se da prioridad tanto a los valores que enfatizan la autonomía de la persona como un ser independiente y socialmente responsable de los demás. Mientras que en Estados Unidos los valores más importantes son la autonomía de la persona 
pero ligado a la búsqueda del éxito y del placer, así como los valores de seguridad y orden social.

En síntesis, ambos modelos, el de Inglehart y el de Schwartz, cubren un universo común de significado y, a su vez, cada uno refleja matices propios. En el modelo de Schwartz se cumplen todas las relaciones de incompatibilidad y el $75 \%$ de las relaciones de compatibilidad postuladas. Dadas las relaciones encontradas entre los valores culturales del Postmaterialismo y los valores culturales de la teoría de Schwartz, a partir de ahora trabajaré sólo con las puntuaciones de los países en Postmaterialismo, Igualitarismo y Autonomía Intelectual y Afectiva.

\section{Antecedentes y consecuencias de los valores culturales}

Para poder abordar esta cuestión tenemos que proceder por pasos. Es cierto que los factores económicos y de desarrollo humano están afectados por un conjunto de factores de los cuales los valores pueden ser o no de escasa importancia. Pero para indagarlo hemos relacionado un conjunto de variables socioeconómicas con las dimensiones culturales. En primer lugar, hemos relacionado las puntuaciones de los países en los valores de Postmaterialismo, de Autonomía Intelectual y Afectiva y de Igualitarismo, de los años 1990, con las puntuaciones de estos mismos países en las variables socioeconómicas de PIB e IDH, de los años 1985. En segundo lugar, hemos relacionado los mismos valores con estas mismas variables socioeconómicas pero esta vez de años posteriores a la medición de los valores culturales, años 95-97. En el primer caso, los valores se consideran consecuentes de las variables sociodemográficas y, en el segundo caso, antecedentes de las mismas.

La tabla 2 muestra en primer lugar la relación entre las variables socioeconómicas como antecedentes de los valores culturales.

\section{TABLA 2}

Correlación entre las variables estructurales de los años 1985

y los valores culturales de los años 1990

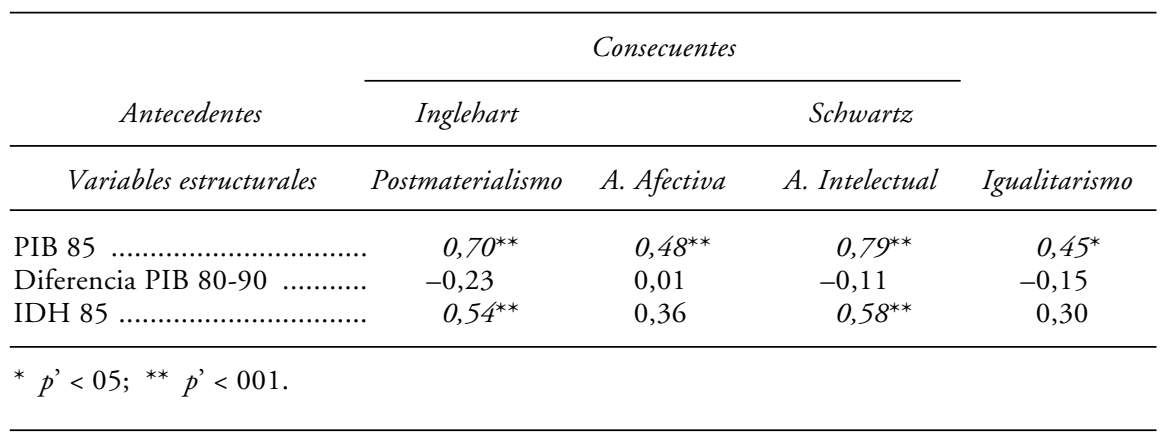


PIB e IDH del 85 son los antecedentes más importantes del Postmaterialismo en los años noventa. Mientras que el nivel de renta per cápita e índice de desarrollo humano del país están positivamente relacionados con el Postmaterialismo, éste se encuentra negativamente relacionado con el crecimiento económico. De forma similar, el PIB es el antecedente más importante de la Autonomía Intelectual, de la Autonomía Afectiva y del Igualitarismo. El IDH es también el antecedente más importante de la Autonomía Intelectual, después de la Autonomía Afectiva y del Igualitarismo.

Por tanto, podemos concluir que estas variables socioestructurales se comportan de forma similar con los cuatro valores culturales analizados. Además, mientras que la riqueza, medida como PIB, es un antecedente del Postmaterialismo, cuando se mide como crecimiento económico está negativamente relacionado con él, tal y como predice la teoría de Inglehart. El crecimiento económico está positivamente relacionado con la importancia de los valores materialistas compatibles con la industrialización. Algo similar le ocurre al PIB y al crecimiento económico con los valores de Autonomía Intelectual, Afectiva e Igualitarismo.

Mientras que el PIB se relaciona con las cuatro dimensiones culturales, el IDH, medido como expectativa de vida, conocimientos (alfabetización de adultos) e ingresos (PIB per cápita), se relaciona sólo con el Postmaterialismo y la Autonomía Intelectual (curiosidad, creatividad y apertura de mente).

También he estudiado la dirección opuesta de influencia, es decir, en qué medida los valores culturales pueden ser responsables de cambios en las condiciones socioeconómicas de los países. Para lo cual he seleccionado las puntuaciones socioeconómicas de estos mismos países, entre cinco y ocho años después de haberse recogido sus puntuaciones en los valores culturales, y las he relacionado entre sí.

\section{TABLA 3}

Correlación entre las variables culturales de los años 1990 $y$ las variables estructurales de los años 1995

\begin{tabular}{|c|c|c|c|c|}
\hline \multirow[b]{2}{*}{ Consecuentes } & \multicolumn{4}{|c|}{ Antecedentes } \\
\hline & Inglehart & & Schwartz & \\
\hline Variables estructurales & Postmaterialismo & A. Afectiva & A. Intelectual & Igualitarismo \\
\hline PIB 95 & $0,82^{* *}$ & $0,68^{* *}$ & $0,78^{* *}$ & $0,66^{* *}$ \\
\hline Diferencia PIB $90-95 \ldots \ldots \ldots \ldots$ & $-0,09$ & 0,17 & 0,16 & 0,20 \\
\hline Export. alta tecnología $97 \ldots .$. & $0,36^{*}$ & 0,34 & 0,07 & 0,07 \\
\hline 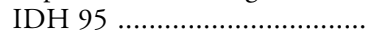 & $0,66^{* *}$ & $0,53^{* *}$ & $0,67^{* *}$ & $0,56^{* *}$ \\
\hline Diferencia IDH $90-95 \ldots \ldots \ldots . .$. & $-0,11$ & 0,25 & $-0,12$ & $0,42^{*}$ \\
\hline
\end{tabular}


Los resultados de la tabla 3 muestran que cuatro valores culturales, Postmaterialismo, Autonomía Intelectual y Afectiva e Igualitarismo, de los años noventa están positivamente relacionados tanto con el PIB como con el IDH, así como con la exportación de alta tecnología, entre los años 1995 y 1997. Por tanto, los valores culturales también parecen antecedentes del PIB del 95 y del IDH del 95. Ahora bien, mientras que los valores postmaterialistas están negativamente relacionados con el crecimiento económico, los valores del Igualitarismo sí están relacionados con el incremento del IDH unos años más tarde.

Por tanto, la conclusión de los datos de las dos tablas anteriores es que tanto las variables socioeconómicas parecen influir en los valores culturales como éstos, a su vez, influyen en el desarrollo de estas condiciones socioeconómicas. Ahora bien, si nos fijamos en la intensidad de las correlaciones en el periodo analizado, la intensidad de la influencia parece algo mayor desde los valores culturales al desarrollo socioeconómico que a la inversa. Sin embargo, sería necesario regresar ambos grupos de variables para ver con mayor precisión si estos resultados se sostienen.

Hemos analizado la importancia relativa de los valores culturales y de la situación económica anterior sobre el desarrollo económico y el IDH. Para lo cual hemos regresado los índices de las puntuaciones culturales de estos 40 países durante los años noventa con el PIB del año 95 y con el IDH del 95. También hemos visto si los cambios económicos y los cambios en el IDH, producidos en un periodo de diez años, están influidos por el nivel económico o de desarrollo humano previo, así como por los valores culturales.

Vamos a ver en primer lugar los resultados del desarrollo económico. Los predictores del desarrollo económico, Autonomía Intelectual y Afectiva, Igualitarismo, Postmaterialismo y PIB 85, están altamente intercorrelacionados. Por tanto, existe un gran solapamiento, lo cual refleja las interconexiones de estos predictores en el mundo real.

Hemos trabajado con dos modelos. Cada uno de ellos incluye por etapas distintas variables. Tal y como podemos ver en la tabla 4, el primero regresa sólo las tres variables culturales de la teoría de Schwartz, Autonomía Intelectual y Afectiva e Igualitarismo.

En el modelo 1, tres variables culturales explican el 78\% de la varianza en el PIB del 95. En el segundo modelo entra, además, el Postmaterialismo y su $\mathrm{R}^{2}$ sube a 87 , lo cual significa que el valor cultural de la teoría de Inglehart añade un 9\% de varianza explicada. En el tercero entra, además, el PIB del 85 y esta variable añade otro $8 \%$ de la varianza, su $\mathrm{R}^{2}$ sube a 95 . Es, por tanto, este tercer modelo el que más varianza explica, hasta el 95\% del PIB del 95. Además, los dos primeros modelos sirven para explicar los factores que influyen en la riqueza de un periodo económico específico, mientras que el tercero permite conocer los antecedentes del cambio económico en un periodo de diez años.

Veamos primero los antecedentes del PIB del año 95. El modelo 2 es el que proporciona la mejor explicación $\left(\mathrm{R}^{2}=87\right)$. El PIB del 95 se explica tanto 


\section{TABLA 4}

Regresión del efecto de variables culturales y PIB 85 sobre PIB del año 95

\begin{tabular}{|c|c|c|c|}
\hline & Modelo 1 & Modelo 2 & Modelo 3 \\
\hline 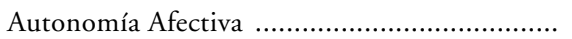 & 0,16 & 0,14 & $0,21^{* *}$ \\
\hline Autonomía Intelectual & $0,54^{* * *}$ & $0,40^{* * *}$ & 0,02 \\
\hline 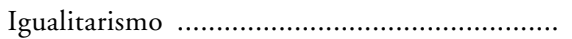 & $0,37^{* *}$ & 0,16 & $0,15^{*}$ \\
\hline Postmaterialismo & & $0,42^{* * *}$ & $0,20^{*}$ \\
\hline PIB 85 & & & $0,58^{* * *}$ \\
\hline $\mathrm{R}^{2}$ & 0,78 & 0,87 & 0,95 \\
\hline
\end{tabular}

${ }^{*} p^{\prime}<0,5 ;^{* *} p^{\prime}<0,01 ;{ }^{* * *} p^{\prime}<0,001$.

Nota: Los datos presentados en esta tabla son coeficientes Beta.

por los valores culturales del Postmaterialismo $\left(\right.$ Beta $\left.=0,42, p^{\prime}<001\right)$ como de la Autonomía Intelectual (Beta $\left.=0,39, p^{\prime}<001\right)$. Por tanto, estos dos valores culturales parecen antecedentes de la riqueza generada cinco años más tarde. Ni la Autonomía Afectiva ni el Igualitarismo parecen tener una contribución significativa a esta riqueza

Veamos ahora, en el modelo 3, el cambio económico entre el 85 y el 95. Este cambio se explica tanto por las condiciones económicas previas, PIB 85 (Beta $\left.=0,58, p^{\prime}<000\right)$, como por los valores del Postmaterialismo $\left(\right.$ Beta $\left.=0,20, p^{\prime}<004\right)$, del Igualitarismo $\left(\right.$ Beta $\left.=0,15, p^{\prime}<006\right)$ y de la Autonomía Afectiva (Beta $\left.=0,21, p^{\prime}<01\right)$. Al cambio económico contribuyen primero el nivel económico previo y, además, un conjunto de valores culturales autoexpresivos, de riesgo, de disfrutar de la vida y de igualitarismo. Contrariamente a lo que Inglehart sugiere, el Postmaterialismo también contribuye a este desarrollo económico, al menos explica el $9 \%$ de la correlación no común entre el PIB del 85 y el PIB del 95.

Estos resultados no cambian si introducimos primero los valores culturales del Postmaterialismo y después los demás predictores.

Veamos ahora los predictores del IDH del 95. También en este caso, todas las variables están bastante interrelacionadas. En la tabla 5 aparecen los resultados de los cuatro modelos analizados.

En el primer modelo entra sólo el Postmaterialismo, que explica el 44\% de la varianza del IDH del 95. En el segundo modelo hemos añadido las tres variables culturales, Autonomía Intelectual y Afectiva e Igualitarismo, y su $\mathrm{R}^{2}$ sube a 60. En el tercero hemos añadido el IDH del 85 y su $\mathrm{R}^{2}$ sube a 96. Finalmente, en el cuarto hemos quitado el Postmaterialismo y su $\mathrm{R}^{2}$ permanece en 96.

Los antecedentes más potentes del IDH del 95 vienen expresados en el modelo 2. La Autonomía Intelectual (Beta $\left.=0,43, p^{\prime}<01\right)$ es el antecedente 


\section{TABLA 5}

Regresión de los valores culturales e IDH del 85 sobre IDH del 95

\begin{tabular}{|c|c|c|c|c|}
\hline & Modelo 1 & Modelo 2 & Modelo 3 & Modelo 4 \\
\hline Postmaterialismo ......................... & $0,67^{* * *}$ & 0,29 & $-0,01$ & \\
\hline Autonomía Intelectual ........... & & $0,43^{*}$ & 0,06 & 0,06 \\
\hline Autonomía Afectiva ................ & & 0,03 & 0,08 & 0,08 \\
\hline 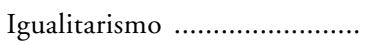 & & 0,20 & $0,20^{* * *}$ & $0,26^{* * *}$ \\
\hline IDH 85 & & & $0,79^{* * *}$ & $0,79^{* * *}$ \\
\hline $\mathrm{R}^{2}$ & 44 & 60 & 96 & 96 \\
\hline
\end{tabular}

${ }^{*} p^{\prime}<0,05$; $^{* *} p^{\prime}<0,01 ;{ }^{* * *} p^{\prime}<0,005$.

Nota: Los datos presentados son coeficientes beta.

más importante, seguido del Postmaterialismo $($ Beta $=0,29)$ y del Igualitarismo $($ Beta $=0,20)$. Es interesante destacar que el cambio del modelo 1 al modelo 2 no sólo consiste en que se incrementa su poder explicativo, sino que el Postmaterialismo disminuye en su capacidad explicativa y, además, su contribución no es significativa.

El cambio del IDH en los últimos diez años se explica mejor por el modelo 3 y, sobre todo, el 4 . En el modelo 3 sólo influye una variable cultural, el Igualitarismo (Beta $\left.=0,20, p^{\prime}<000\right)$, y, además, el IDH del 85 (Beta $=0,79, p^{\prime}<$ 000) es el antecedente más potente. Por tanto, el cambio en el IDH se explica fundamentalmente por el nivel previo de IDH y por la importancia que se otorga a los valores que enfatizan la igualdad entre los seres humanos, la libertad, la justicia social, la honestidad y un mundo en paz. Al quitar el Postmaterialismo en el modelo 4 , su $\mathrm{R}^{2}$ permanece idéntico al del modelo 3, mientras que mejora el poder predictivo del Igualitarismo.

En resumen, se observan una serie de factores comunes en las teorías en la explicación del PIB y del IDH del 85. Las dimensiones culturales en ambos casos explican una parte importante de estas variables dependientes, casi la tercera parte en el caso del desarrollo humano y casi la mitad del producto interior bruto. Sin embargo, también se observan aspectos discriminantes: por ejemplo, las variables culturales de Autonomía (Intelectual y Afectiva) e Igualitarismo explican más del PIB 85 que el Postmaterialismo, mientras que el Igualitarismo explica más del IDH del 95 que el Postmaterialismo.

Cuando analizamos el cambio económico o de desarrollo humano en la década del 85-95, la validez convergente de ambas teorías se observa en que ambas contribuyen — detrás del PIB previo y del IDH previo- a explicar el cambio tanto en las condiciones económicas como en el desarrollo humano en esta década. Su validez discriminante consiste en que mientras que las variables culturales de la teoría de Schwartz contribuyen a explicar tanto el cambio eco- 
nómico como el cambio en el desarrollo humano, el Postmaterialismo sólo contribuye a explicar el cambio económico. Por lo tanto, contrariamente a lo postulado por Inglehart, el Postmaterialismo también contribuye a explicar el cambio económico.

\section{CONCLUSIONES}

Este trabajo ha realizado una comparación de las teorías culturales de Inglehart y Schwartz siguiendo dos procedimientos. Uno ha consistido en analizar sus similitudes y diferencias correlacionando sus puntuaciones en las dimensiones culturales y en los mismos países estudiados por sus autores. El otro procedimiento ha consistido en comparar el peso relativo de cada una de las dimensiones culturales junto a otros factores macrosociales y macroeconómicos en su contribución a la riqueza y al crecimiento económico de los países, así como a su desarrollo y crecimiento social.

Al hacerlo he encontrado que las similitudes conceptuales entre las dos teorías contrastadas se producen entre las dimensiones culturales del Postmaterialismo de Inglehart y la Autonomía Intelectual y Afectiva y el Igualitarismo de Schwartz. Por lo tanto, las diferencias de nomenclatura entre las dos teorías en estas dimensiones esconden un significado compartido. Así, cuando Inglehart habla de la dimensión cultural de Postmaterialismo está refiriéndose a los mismos valores que Schwartz cuando alude a las dimensiones culturales de énfasis en la Autonomía de la persona y de Compromiso igualitario. Este resultado es aún más robusto cuando consideramos otras diferencias entre ambas teorías en muestras e instrumentos de medición, o incluso en los factores responsables de la puntuación de los países en estas dimensiones culturales.

Ahora bien, también se observan diferencias entre ambas. Las mayores diferencias se observan entre el Postmaterialismo y las dimensiones culturales de Conservación, Jerarquía y Competencia. Estas diferencias van en sentido opuesto. Es decir, que ser alto en la dimensión cultural de Postmaterialismo significa también ser bajo en las dimensiones culturales de Conservación, Jerarquía y Competencia. En contra de lo pronosticado, el Postmaterialismo no está relacionado con la Armonía, debido a que la preocupación por el medio ambiente, presente en la dimensión cultural de Schwartz, está ausente de la dimensión cultural de Inglehart,debido a su falta de identidad de significado entre las culturas (Inglehart, 1998).

En síntesis, la diferencia entre el modelo unidimensional de Inglehart ${ }^{6}$ y el bidimensional de Schwartz consiste en que el modelo bidimensional aporta

${ }^{6}$ Es necesario señalar que los datos correspondientes a la segunda dimensión cultural de Inglehart no estaban publicados en el momento de realizarse este trabajo y, por tanto, no han podido ser considerados en este estudio. 
matices que el otro no percibe. Uno de estos matices está en la relación entre la Autonomía Intelectual y Afectiva y el Igualitarismo, que implica la posibilidad de encontrar un individualismo que trata de desarrollar la autonomía de la persona junto a un colectivismo que enfatiza la preocupación por el bienestar de los demás. Esto implica superar la vieja antinomia individualismo-colectivismo, presente en la teoría social en dos sentidos. En un sentido, individualismo no sólo significa egoísmo, sino también autonomía de la acción, y colectivismo no sólo significa solidaridad, sino también conservación y tradición. $\mathrm{Y}$, en el otro, es posible encontrar juntas y, por tanto, dándose a la vez la autonomía de la acción y la interdependencia y la reciprocidad. Estos resultados contradicen hipótesis más pesimistas que caracterizan el individualismo contemporáneo de las sociedades desarrolladas sólo como narcisista (Béjar, 1993) y permiten dar una visión más positiva y matizada del mismo. A la vez, refuerzan algunas de las soluciones propuestas por Beck (1992) a la sociedad del riesgo que se centran en la transparencia democrática. Según este autor, una opinión pública vigorosa - ilustrada y reflexiva- constituye el mejor antídoto contra la incertidumbre. Los psicólogos sociales comprometidos con los valores de autorrealización deberían ser conscientes de que es posible actualizar el desarrollo de la persona como agente capaz de desarrollar metas propias junto a los valores de compromiso igualitario. La traducción práctica de esta conclusión es que estos aspectos culturales deberían tenerse en cuenta en el desarrollo de los contenidos educativos y en la forma de estructurar y evaluar los contextos organizacionales.

En segundo lugar, los valores son resultado de una serie de condiciones macroeconómicas. Siguiendo la teoría funcionalista, los valores que se refuerzan son los que son compatibles con una forma determinada de funcionamiento social. Así, mientras que el crecimiento económico refuerza los valores materiales, el desarrollo humano refuerza valores más solidarios. Ahora bien, la orientación de valores explica gran parte del desarrollo económico y del desarrollo humano posterior. Lo cual refleja la influencia de las personas como agentes sociales activos en la creación de condiciones socioeconómicas determinadas y en la construcción social en general. En síntesis, el carácter intencional y socialmente compartido de la búsqueda de cambios ha sido el distintivo de los movimientos colectivos. Según Laraña y Gusfield (1994), estos movimientos son actualmente expresión tanto de la ideología como de la identidad social. Representan signos de que se están cuestionando determinados aspectos de la vida social y de que hay otras alternativas. Así, los movimientos contemporáneos a favor de la igualdad de género, de un trato no xenófobo hacia los inmigrantes o de respeto hacia los homosexuales, se pueden caracterizar por compartir los valores de igualdad y tolerancia junto a los valores de autoexpresión y autodesarrollo de la persona. Otros, como los movimientos étnicos de exclusión, xenófobos o nacionalistas, parecen compartir los valores de conservación y de jerarquía. Y otros, como los movimientos verdes o ecologistas o los movimientos a favor de la paz, parecen reflejar 
los valores de armonía con el entorno y con las relaciones humanas. Estos movimientos colectivos tienen como finalidad influir en la opinión pública, en las decisiones políticas y, en último término, en las condiciones socioestructurales.

Frente a explicaciones polarizadas en términos de estructura o de autonomía de la acción social, este trabajo apoya una integración de las dos explicaciones. En línea con el concepto de interdependencia de Elias (1990) y con la teoría de la estructuración de Giddens (1967/87), la estructura, en este caso socioeconómica, deja de tener un contenido determinista para integrar los resultados de la acción intencional, es decir, de los valores. De este modo creemos haber contribuido, siguiendo la sugerencia de R. Turner (1988), a reequilibrar en la teorización psicosociológica el análisis de cómo los individuos afectan la estructura y procesos de la sociedad.

Es cierto, sin embargo, que los efectos de las estructuras sociales sobre las personas son más rápidos y consistentes, mientras que los de las personas sobre las estructuras sociales son más lentos y a más largo plazo, tal y como han demostrado Schooler (1996) y Khon y col. (1997) en sus análisis intercultural de los efectos mutuos de la estructura ocupacional y de la personalidad. Es, por tanto, una limitación de este estudio no haber podido analizar el impacto de los valores a más largo plazo, ya que el tiempo transcurrido desde la medición de los valores culturales y su impacto sobre las estructuras socioeconómicas se ha tenido que limitar, por razones obvias, a sólo una década. Sería deseable repetir estos análisis dentro de un lustro o una década porque, siguiendo la teoría actual, es esperable que el impacto de la acción intencional sobre las estructuras sociales fuese aún mayor.

\section{REFERENCIAS}

BELL, D. (1973): El advenimiento de la sociedad postindustrial, Madrid, Alianza.

BÉJAR, H. (1993): La Cultura del Yo, Madrid, Alianza Edit.

BECK, U. (1992): Risk Society, Londres, Sage.

Diener, E., y Diener, C. (1995a): "The wealth of nations revisited: Income and quality of life, Social Indicators Research, 36, 275-286.

DíEZ Nicolás, J. (1994): "Postmaterialismo y desarrollo económico en España», en Juan Díez Nicolás y Ronald Inglehart (eds.), Tendencias mundiales de cambio en los valores sociales y politicos: Una perspectiva global, Madrid, Fundesco, pp. 125-156.

ElIAS, N. (1990): La sociedad de los individuos, Barcelona, Edicions 62.

Giddens, A. (1967/87): Las nuevas reglas del método sociológico, Buenos Aires, Amorrortu.

Gouveia, V., y Ros, M. (2000): «The Hofstede and Schwartz models for classifying individualism at the cultural level: Their relation to macrosocial and macroeconomic variables», Psicothema, 12, 25-33.

Hofstede, G. (1980): Culture's Consequences: International Differences in Work related Values, Beverly Hills (CA), Sage.

- (1991): Cultures and Organizations, software of the mind, Great Britain, MacGraw-Hill.

Human Development Report (1990), Oxford, Oxford University Press.

Informe sobre el Desarrollo Humano (1998), Madrid, Mundi Prensa. 
Inglehart, R. (1998): Modernización y Postmodernización: El cambio cultural, económico y politico en 43 sociedades, Madrid, CIS

INGLEHART, R., y BAKER, W. (2000): «Modernization, cultural change and the persistence of traditional values", American Sociological Review, vol. 65, 19-51.

Inglehart, R., y Klingeman, H. (2000): «Genes, Culture, Democracy and Happiness», en Ed Diener y Eunkook M. Suh (eds.), Culture and subjective well-being, Cambridge, The MIT Press.

InKelEs, A., y SMITH, P. (1974): Becoming Modern: individual changes in six developing countries, Cambridge (MA), Harvard University Press.

KLUCKHÖN, C. (1951): «Value and value orientations in the theory of action», en T. Parsons y E. Shils (eds.), Toward a general theory of action, Cambridge (MA), Harvard University Press.

Kim, U.; Triandis, H. C.; Kagitcibasi, C.; Choi, S.-C., y Yonn, G. (eds.) (1994): Individualism and Collectivism: Theory, method and applications, London, Sage.

Kohn, M. L.; Slomczynski, K. M.; Janicka, M.; Khmelko, V.; Mach, B. W.; Paniotto, V.; Zaborowski, W.; Gutiérrez, R., y Heyman, C. (1997): "Class, work and personality under conditions of radical social change», Annual Review of Sociology, 62, 614-638.

LARAÑA, E., y GUSFIELD, J. (1994): Los nuevos movimientos sociales: de la ideología a la identidad, Madrid, CIS.

MacClelland, D. C. (1961): The achieving society, Princeton (NJ), Van Nostram.

Ros, M., y SCHWARTZ, Sh. (1995): "Jerarquía de valores en países de Europa Occidental: una comparación transcultural», Revista Española de Investigaciones Sociológicas, 69, 69-88.

SCHOOLER, C. (1996): «Cultural and socio-structural explanations of cross-national psychological differences", Annual Review of Sociology, 22, 323-349.

SCHWARTZ, Sh. (1992): "Universals in the content and structure of values: Theoretical advances and empirical tests in 20 countries», en M. Zanna (ed.), Advances in Experimental Social Psychology, vol. 25 (1-65), New York, Academic Press

- (1994): «Are there universals aspects in the structure and contents of human values?, Journal of Social Issues, 50, 19-45.

- (1999): «A theory of cultural values and some implications for work», Applied Psychology: An International Review, 48, 1, 23-47.

SChWARTZ, Sh., y Melach, G. (2000): «National differences in micro and macro worry: Social, economic and cultural explanation», en Ed Diener y Eunkook M. Suh (eds.), Culture and subjective well-being, Cambridge, The MIT Press.

SCHWARTZ, Sh., y Ros, M. (1996): "Values in the West: A theoretical and empirical challenge to the Individualism-Collectivism dimension», World Psychology, 1 (2), 91-122.

TRIANDis, H. (1990): «Cross-cultural studies of individualism and collectivism», en J. Berman (ed.), Nebraska Symposium of Motivation, 1989, Lincoln, University of Nebraka Press, pp. 41-133.

- (1996): "The psychological measurement of cultural syndromes», American Psychologist, 51, 407-415.

Triandis, H., y Gelfand, M. J. (1998): «Converging Measurement of Horizontal and Vertical Individualism and Collectivism", Journal of Personality and Social Psychology, 74, 118-128.

TURner, R. (1988): «Personality in Society: Social Psychology contribution to Sociology», Social Psychological Quarterly, 51, 1, 1-10. 


\begin{abstract}
At present, the transcultural theories of Hofstede (1980), Schwartz (1944), Triandis (1995) and Inglehart (1998) represent the most confirmed formulations on the cultural dimensions of values. Despite the interest and popularity of Inglehart's model, there are no papers that attempt to analyse the convergent, discriminatory validity of this theory in relation to other cultural theories. This paper attempts to fill the gap by comparing the dimension of Inglehart's postmaterialism with Schwartz's cultural dimensions of autonomy, conversation, hierarchy, egalitarianism, harmony and competence. In order to do this, we follow several processes. To begin with, we study the relationship between the scores in the cultural dimensions of both models with a sample of 40 countries. Then we list the convergent cultural dimensions with external, macrosocial and macroeconomic variables of these societies. Lastly, we analyse the impact of culture, measured by values, and the previous socio-economic standard on the economic and social development of those same societies. The results indicate that the conceptual similarity between the two theories occurs between the cultural dimensions of postmaterialism, intellectual and affective autonomy and egalitarianism; that the values are the result of a series of macroeconomic and macrosocial conditions. And so, while economic growth reinforces material values, human development reinforces values that are more shared. However, the orientation of values explains a large part of economic development and subsequent human development.
\end{abstract}

